\title{
Lipid Laden Macrophages in Respiratory Disease
}

TO THE EDITOR: Studies using the lipid laden macrophage index (LLAMI), first described by Colombo and Hallberg, ${ }^{1}$ have shown an unquestionable excess of lipid laden macrophages in the airways of patients with respiratory diseases. ${ }^{1,2}$ However, the diagnostic use of the LLAMI to detect reflux and aspiration of gastric contents is still debated. Varying reports have used differing patient inclusion/exclusion criteria and differing methods of gastroesophageal reflux disease diagnosis, which may contribute to the lack of specificity. The recent paper by Özdemir et al $l^{3}$ claims that LLAMI is a useful diagnostic tool for detecting microaspiration in patients with chronic cough, heartburn, and physiological evidence of acid reflux on esophageal $\mathrm{pH}$ monitoring.

We undertook a study with patients with a variety of respiratory diseases and compared those with a history of airway reflux, as confirmed by the Hull Airway Reflux Questionnaire (HARQ). ${ }^{4}$ Using surplus bronchoalveolar lavage fluid, cells were stained with
Oil Red $\mathrm{O}$ and quantified using the LLAMI (Fig. 1).

A total of 29 patients consented to allow surplus bronchoalveolar lavage fluid to be stained by Oil Red O. Patient data were collected and anonymised. Patients were categorised by a blinded observer following a review of clinical records. Those with a diagnosis of reflux disease $(\mathrm{n}=15)$ had a mean HARQ score of 28.6, mean age of 62.5 , and a female preponderance of $57.1 \%$, whereas those without diagnosis of reflux $(n=14)$ had a HARQ score mean of 12.5 , mean age 63.9 , and $78.6 \%$ female.

Despite using, in our opinion, a more accurate diagnostic tool for airway reflux, the HARQ score, there was no significant correlation between LLAMI and reflux diagnosis (non-parametric Mann-Whitney test, $P=0.910$; Fig. 2). In contrast to our study, which comprised of general respiratory patients, Özdemir's patients were those with acid reflux disease and respiratory symptoms. We conclude that macrophages are not only capable of accumulating
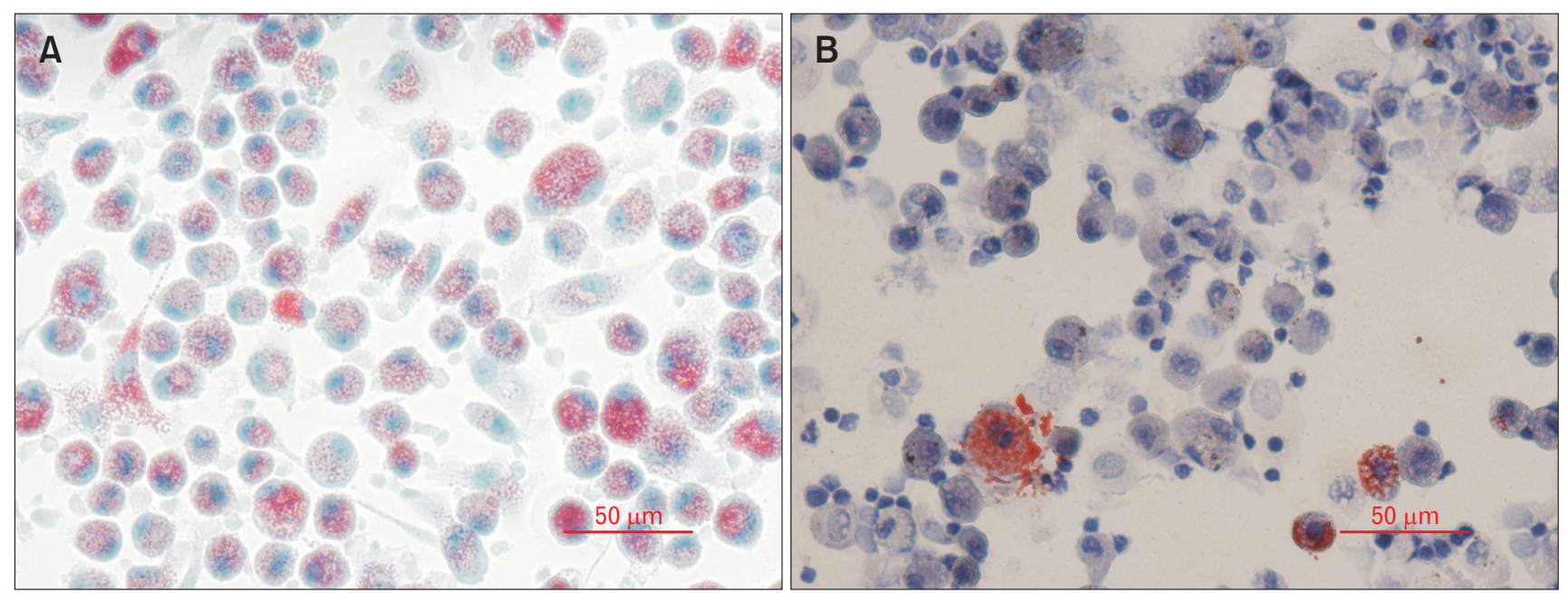

Figure 1. Primary alveolar macrophages stained with Oil Red $\mathrm{O}$ and Hematoxylin. Bronchoalveolar lavage samples were processed and Cytospun onto glass slides. Cells were stained for lipids using Oil Red O and counterstained with Mayer's Hematoxylin. Lipid droplets stained vivid red and were easily distinguishable against the purple counterstain. Patients showed a high degree of variability of lipid accumulation. Patient (A) shows many cells with lipid accumulation whilst patient (B) shows only a few cells with lipid accumulation (bar $=50 \mu \mathrm{m}$ ). 


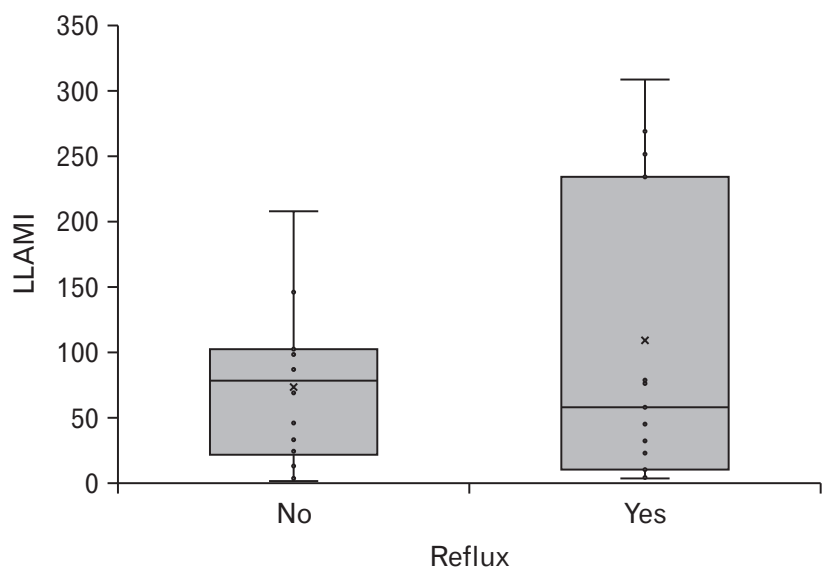

Figure 2. Comparison of means between lipid laden macrophage index (LLAMI) and gastroesophageal reflux diagnosis. Box plot to show comparison of means between patient LLAMI scores and patients diagnosed with reflux. Significance $(P)=0.910$.

lipids directly from food material, ${ }^{5}$ but can also accumulate lipids from the phagocytosis of other lipid containing cells ${ }^{6}$ which may not necessarily be available solely in the reflux associated airway pathology.

Laura R Sadofsky, Yvette A Hayman,
Simon P Hart, and Alyn H Morice

Center for Cardiovascular and Metabolic Research, Hull York Medical School, The University of Hull, Castle Hill Hospital, Cottingham, UK
1. Colombo JL, Hallberg TK. Recurrent aspiration in children: lipid-laden alveolar macrophage quantitation. Pediatr Pulmonol 1987;3:86-89.

2. Lahiri $\mathrm{T}$. The utility of the lipid-laden macrophage index for the evaluation of aspiration in children. Cancer Cytopathol 2014;122:161-162.

3. Özdemir P, Erdinç M, Vardar R, et al. The role of microaspiration in the pathogenesis of gastroesophageal reflux-related chronic cough. J Neurogastroenterol Motil 2017;23:41-48.

4. Morice AH, Faruqi S, Wright CE, Thompson R, Bland JM. Cough hypersensitivity syndrome: a distinct clinical entity. Lung 2011;189:7379.

5. Hayman Y, Sadofsky LR, Williamson JD, Hart SP, Morice AH. The effects of exogenous lipid on THP-1 cells: An in vitro model of airway aspiration? ERJ Open Res Published Online First: 20 Mar 2017. doi: 10.1183/23120541.00026-2016.

6. Nakahira K, Pabon Porras MA, Choi AM. Autophagy in Pulmonary Diseases. Am J Respir Crit Care Med 2016;194:1196-1207.

\section{Conflicts of interest: None.}

Author contributions: Laura R Sadofsky: interpreting the data and drafting the manuscript; Yvette A Hayman: conducting the study, collecting and interpreting the data, proof reading the manuscript; Simon P Hart: conception and design, data interpretation, and revising the article; Alyn $\mathrm{H}$ Morice: conception and design, revising the manuscript, and interpretation of the data; and all authors approved the final version. 